\title{
Automated angular and translational tomographic alignment and application to phase- contrast imaging
}

\author{
Cunha Ramos, Tiago Joao; Jørgensen, Jakob Sauer; Andreasen, Jens Wenzel
}

Published in:

Journal of the Optical Society of America A

Link to article, DOI:

10.1364/JOSAA.34.001830

Publication date:

2017

Document Version

Peer reviewed version

Link back to DTU Orbit

Citation (APA):

Cunha Ramos, T. J., Jørgensen, J. S., \& Andreasen, J. W. (2017). Automated angular and translational tomographic alignment and application to phase-contrast imaging. Journal of the Optical Society of America A, 34(10), 1830-1843. https://doi.org/10.1364/JOSAA.34.001830

\section{General rights}

Copyright and moral rights for the publications made accessible in the public portal are retained by the authors and/or other copyright owners and it is a condition of accessing publications that users recognise and abide by the legal requirements associated with these rights.

- Users may download and print one copy of any publication from the public portal for the purpose of private study or research.

- You may not further distribute the material or use it for any profit-making activity or commercial gain

- You may freely distribute the URL identifying the publication in the public portal 


\title{
Automated angular and translational tomographic alignment and application to phase-contrast imaging
}

\author{
T. RAMOS, ${ }^{1}$ J. S. JøRGENSEN, ${ }^{2}$ J. W. ANDREASEN ${ }^{1 *}$ \\ ${ }^{1}$ Technical University of Denmark, Department of Energy Conversion and Storage, Frederiksborgvej 399, 4000 Roskilde, Denmark \\ ${ }^{2}$ Technical University of Denmark, Department of Applied Mathematics and Computer Science, Richard Petersens Plads, \\ Building 324, 2800 Kongens Lyngby, Denmark \\ *Corresponding author: jewa@dtu.dk
}

\begin{abstract}
X-ray Computerized Tomography (CT) is a 3D imaging technique that makes use of X-ray illumination and image reconstruction techniques to reproduce the internal cross sections of a sample. Tomographic projection data usually requires an initial relative alignment or knowledge of the exact object position and orientation with respect to the detector. As tomographic imaging reaches increasingly better resolution, thermal drifts, mechanical instabilities and equipment limitations are becoming the main dominant factors contributing to sample positioning uncertainties that will further introduce reconstruction artifacts and limit the attained resolution in the final tomographic reconstruction. Alignment algorithms that require manual interaction impede data analysis with everincreasing data acquisition rates, supplied by more brilliant sources. We present in this paper an iterative reconstruction algorithm for wrapped phase projection data and an alignment algorithm that automatically takes 5 degrees of freedom, including the possible linear and angular motion errors, into consideration. The presented concepts are applied to simulated and real measured phase-contrast data, exhibiting a possible improvement in the reconstruction resolution. A MATLAB implementation is made publically available under the DOI: 10.5281/zenodo.495122, and will allow robust analysis of large volumes of phase-contrast tomography data. (C) 2017 Optical Society of America
\end{abstract}

OCIS codes: (340.0340) X-ray optics; (100.6890) Three-dimensional image processing; (100.6950) Tomographic image processing; (110.6960) Tomography; (170.7440) X-ray imaging; (340.6720) Synchrotron radiation;

http://doi.org/10.1364/JOSAA.34.001830

\section{INTRODUCTION}

X-ray Computerized Tomography (CT) is a non-destructive imaging technique that makes use of X-ray transmission projections and tomographic reconstruction algorithms to reproduce the internal refractive properties of a sample.

X-ray projection data can be acquired by different techniques with different contrast modes e.g., by X-ray absorption or coherent diffraction imaging (CDI) as long as the different components that constitute the sample display different interactions with the incident beam and the effect increases linearly over the sample thickness along the beam direction. X-ray CDI does not rely on Xray lenses nor optics and thus has the potential for spatial resolution comparable to the X-ray wavelength, with demonstrated best resolution of $5 \mathrm{~nm}$ in 3D [1]. Ptychography is a variant of the CDI technique in which multiple diffraction measurements from overlapped regions can be used to extend the illuminated field of view to areas much larger than the illumination probe. The best demonstrated spatial resolution with ptychography is currently in the $8-10 \mathrm{~nm}$ range in 2D [2]-[4]. Holler et al. [5] further demonstrated that this imaging technique can be used to reach an isotropic 3D resolution of $15 \mathrm{~nm}$ in a tomographic reconstructed volume.

Three-dimensional phase-contrast imaging usually comprises three different steps: phase retrieval, tomographic alignment and tomographic reconstruction.
Iterative phase retrieval algorithms make use of a single full-field diffraction pattern, or several overlapping scans (ptychography), to recover a complex-valued image of the transmitted radiation over the illuminated sample. The properties inherent to such recovered projection images are further described in section 1.1. Tomographic alignment refers to the determination of the relative sample-detector orientation for each projection image. Tomographic reconstruction is the computational procedure that returns a three-dimensional representation of the sample from a finite number of two-dimensional transmission projections.

Regardless of the technique used for data acquisition, tomographic reconstruction often requires an initial tomographic alignment operation in order to reconstruct an accurate threedimensional representation of the imaged sample. As the sample projections reach increasingly better resolution, mechanical instabilities or equipment limitations are becoming the main dominant factors contributing to the sample positioning uncertainties that will further introduce reconstruction artifacts and limit the attained resolution in the final tomographic reconstruction.

Different tomographic alignment algorithms have been proposed in the literature with various fundamental principles. These can rely on the use and track of fiducial markers introduced in the sample [6], [7], or on other alignment methods based on the center-of-mass properties of the projection images [8], [9] or on cross-correlation measurements [10]. To these we may add other 
markerless alignment methods that make use of iterative optimization algorithms [11], where one minimizes the disagreement between the measured projections and those synthetically produced from the reconstructed volume [12]-[15]. Nevertheless, these are usually user dependent, relying on visual inspection and manual interaction, they operate slice by slice through the illuminated volume, therefore only optimizing a limited number of degrees of freedom, not taking into consideration all the possible tilts and angular uncertainties that might be present in the experimental setup [16].

The full computational tool described here was initially developed for phase-contrast tomography but can in principle be applied to any other projection data acquired with a parallel-beam setup. A brief description of phase-contrast data is now presented in order to introduce two of the properties that characterize it and that should be taken into account during tomographic alignment and reconstruction: phase wrapping and phase ramps.

\subsection{PHASE-CONTRAST IMAGING}

Imaging methods that exploit the refractive properties of a sample are usually referred to as phase-contrast imaging [17]. These, also known as coherent diffractive or inverse imaging methods, of which ptychography is an example, are lensless optical techniques that make use of coherent light interaction with matter creating diffraction patterns that depend on the refraction properties of the material. For a monochromatic beam and at the far-field (or Fraunhofer) plane, the Helmholtz equation for light wave propagation states that the acquired diffraction patterns may be approximated by the Fourier transform amplitude of the incident wavefront after light-sample interaction. Phase-retrieval algorithms [18] and others as described in [19]-[24] are able to retrieve the reciprocal-space phase information and recover a complex-valued image $O(x, y)$ related to the sample transmissivity $T(x, y)$ by

$$
O(x, y)=T(x, y) \exp (\mathbf{i}(a x+b y+c)) .
$$

Here $x$ and $y$ are the coordinates in the detector coordinate system (as seen in Figure 1), for a single projection image. The realvalued coefficients $a, b$ and $c$ define linear and constant phase terms that we will refer to collectively as phase ramp and arise from ambiguities during the reciprocal-space phase-retrieval operation and uncertainties in the relative beam-detector position. Under the "thin sample" condition [25], the complex transmission function $T(x, y)$ is defined as

$$
T(x, y)=\exp (-B(x, y)) \exp (\mathbf{i} P(x, y)),
$$

where $B(x, y)$ and $P(x, y)$ are defined as

$$
\begin{aligned}
& B(x, y)=\frac{2 \pi}{\lambda} \int \beta(x, y, z) \mathrm{d} z, \\
& P(x, y)=-\frac{2 \pi}{\lambda} \int \delta(x, y, z) \mathrm{d} z,
\end{aligned}
$$

and are known as absorption projection and phase-shift or phasecontrast projection images, respectively. It is worth noticing that (3) and (4) are limited to the "thin sample" condition, where wave propagation effects through the thickness of the sample are not considered [25]. Here $\lambda$ represents the wavelength of the incoming $\mathrm{X}$-ray beam, whereas $\delta$ and $\beta$ are the real and imaginary deviations of the complex refractive index $n$ from unity [17], [26]:

$$
n(x, y, z)=1-\delta(x, y, z)+\mathbf{i} \beta(x, y, z) \text {. }
$$

Three-dimensional tomographic reconstructions from absorption projection and phase-contrast projection images provide a quantitative measure of the refractive index of the materials that constitute the sample under study. Tomographic reconstructions from absorption projections return the imaginary part of the sample complex refractive index in (5) which is associated with its linear absorption coefficient $\mu$ through $\beta=\lambda \mu /(4 \pi)$. On the other hand, reconstructions from phase-contrast projections are directly related to the sample electron density $\rho$ by

$$
\delta(x, y, z)=\frac{\rho(x, y, z) r_{0} \lambda^{2}}{2 \pi},
$$

where $r_{0}=2.82 \cdot 10^{-5} \AA$ is the Thomson scattering length.

Phase-contrast projections provide quantitative data that are usually of higher resolution when compared with their respective absorption projection images, but phase-contrast projections may present additional challenges that must be taken into account prior to the tomographic alignment and reconstruction operation [22], [23], [27]. The two main issues addressed here are the presence of background gradients in the projections that take the form of phase ramps and wrapped areas that are characteristic of coherent diffraction imaging techniques.

Let us define $\bar{P}(x, y)$ as the phase of $O(x, y) . \bar{P}(x, y)$ is commonly computed as $\bar{P}(x, y)=\arg (O(x, y))$ and can be seen as a background-corrupted phase-contrast projection given by

$$
\arg (O(x, y))=\bar{P}(x, y)=P(x, y)+a x+b y+c .(7)
$$

We will refer to $\bar{P}(x, y)$ as non-normalized phase-contrast projection data. The influence of the term $a x+b y+c$ in (7) must be subtracted from $\bar{P}(x, y)$ in order to obtain $P(x, y)$ that will be further used for tomographic reconstruction. We will refer to this constant and linear phase term removal operation as phase ramp removal.

When measuring thick or high-contrast samples phase shifts larger than the wave-front period $(2 \pi)$ may occur. Because phaseretrieval algorithms are insensitive to the periodic nature of phase signals, they only allow the determination of phase values modulo $2 \pi$ commonly represented in $]-\pi, \pi]$ ranges. Phase-projection images can therefore present a wrapping behavior in the pixels associated with phase shift outside the $]-\pi, \pi]$ interval.

With ever-increasing data-acquisition rates, provided by more brilliant synchrotron sources, manual user interaction during phase-ramp removal and tomographic alignment impedes phasecontrast data processing and analysis. This motivates our development of a fully automated alignment and reconstruction algorithm that takes into consideration the presence of phase ramps, phase wrapping and any possible sample-detector relative orientation corrections.

Although the proposed method, described in this paper, could be used for any parallel-beam geometry tomography setup, additional requirements and assumptions are necessary for the proposed algorithm to return accurate reconstructions when phase-contrast data is used. These include a high signal to noise ratio in the acquired data, a maximum translation between two consecutive projections of less than half of their field of view, and a maximum difference between the true and wrapped phase image of $2 \pi$. We have seen these requirements to be fulfilled for most of the acquired phase-contrast tomography data acquired by our team during the last 3 years. A few exceptions to these conditions were observed when analyzing thick and heavy samples where 
wrapping in the phase-contrast projections exceeded more than a full phase period $(2 \pi)$.

Both phase ramps and phase wrapping constitute two immediate obstacles for center-of-mass alignment algorithms. This is mainly due to the fact that center-of-mass alignment methods rely on measurement of the projection pixel intensity values that can be over- or underestimated in the presence of background phase ramps and wrapping. Guizar-Sicairos et al. proposed an alignment method [27] where phase ramps are initially corrected, the projections unwrapped, and a mass-based method is applied in a well-behaved area containing no unwrapping artifacts. Phase unwrapping can be performed by using a 2D Goldstein unwrapping technique [28] or other methods described in the literature [29], [30] but these can sometimes be time-consuming, semi-assisted or introduce undesired artifacts in the projection images that would eventually propagate to the reconstructed volume itself. In the same work published in [27] Guizar-Sicairos et al. introduced a reconstruction method that allows tomographic reconstruction without previous phase unwrapping. The full potential of this method is not realized however, unless it is combined with alignment tools that are equally insensitive to phase wrapping. This has motivated our development of alignment and reconstruction methods that effectively handle effects of phase wrapping, and have the ability to consider any possible sample-detector relative orientation with all the degrees of freedom represented in Figure 1. We characterize our proposed method as fully automated because it functions without any user supervision after an initial set of parameters is defined.

\subsection{CONTRIBUTIONS AND ORGANIZATION OF THIS ARTICLE}

Facing the previously described challenges of phase-contrast imaging, a robust and automated alignment and reconstruction algorithm should:

1. Handle phase wrapping during alignment and reconstruction.

2. Correct for phase ramps.

3. Correct for all the possible linear and angular positioning errors manifested in sample-detector relative position.

In the following Methodology section we present our proposed alignment and reconstruction methods for phase-contrast projection data. The different subsections describe each step of the tomographic alignment and reconstruction algorithms, for which we describe our specific contributions, and are structured as follows:

\footnotetext{
1. Tomographic reconstruction of phase-contrast data.

2. Coarse Translation Alignment (CTA).

3. Phase Ramp Removal (PRR).

4. Fine Projection Alignment (FPA).
}

Finally, the results of the proposed alignment and reconstruction methods are shown for a 3D modified Shepp-Logan phantom simulation and for a real ptychographic dataset acquired at the cSAXS beamline at the Paul Scherrer Institut (PSI), Switzerland. The last comprises a sample of a nanoporous glass overlaid with a thin film of $\mathrm{Ta}_{2} \mathrm{O}_{5}$, already analyzed and published in reference [31] exhibiting an isotropic and three-dimensional resolution of $16 \mathrm{~nm}$. A total of 720 tomographic projections were acquired with equally spaced angles over a 180-degrees angular span. Each projection image was reconstructed from a series of 343 diffraction patterns acquired over an area of $9 \times 3 \mu^{2}$, arranged in concentric circles with $250 \mathrm{~nm}$ radial step-size. Each diffraction pattern was measured at $2.363 \mathrm{~m}$ from the sample with a Pilatus 2M detector (pixel size of $172 \mu \mathrm{m}$ ) using an X-ray beam, $600 \mathrm{~nm}$ in diameter and a photon energy of $6.2 \mathrm{keV}$, with an exposure time of $0.6 \mathrm{~s}$.

\section{NOMENCLATURE AND FORWARD MODEL}

The system of simultaneous linear equations that describe the forward projection procedure can be expressed as

$$
\mathbf{A}(\Theta) x=b,
$$

where $\boldsymbol{b}$ and $\boldsymbol{x}$ are vector forms of the projections and volume reconstruction respectively, and $\mathbf{A}$ is the system matrix that performs the discretized Radon transform. The system matrix acts as a forward operator performing linear discrete integrations through the reconstructed volume $\boldsymbol{x}$ according to specific spatial relative orientations defined by the set of projection parameters $\boldsymbol{\Theta}$.

Our studies were conducted in a parallel-beam geometry setup, as it is characteristic for X-ray ptychography. We assume that the incident beam is perfectly perpendicular to the detector plane with the consequence that sample movements in the incident beam direction have no significant influence on the acquired projections. According to this assumption, there are 5 degrees of freedom (DOF), or independent motions that the sample may experience during the acquisition of tomographic projections. These include 2 linear parameters $u$ and $v$ (horizontal and vertical translation) and 3 angular parameters: the projection angle $\theta$ and the tilt parameters $\alpha$ and $\beta$, see Figure 1.

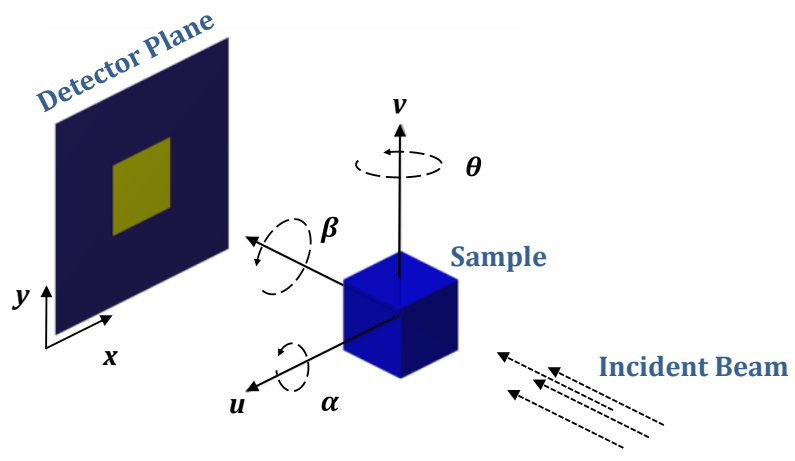

Figure 1: Parallel-beam experimental setup with 5 degrees of freedom.

In the following sections we will refer to these parameters in their vector form including their respective values for all the acquired projections. These vectors are represented in boldface and may also be grouped in a single vector $\boldsymbol{\Theta}=[\boldsymbol{\theta}, \boldsymbol{u}, \boldsymbol{v}, \boldsymbol{\alpha}, \boldsymbol{\beta}]^{\mathrm{T}}$ containing all projection parameters for all the acquired projections. The superscripts 'MEA', 'CTA' and 'FPA' will be used to differentiate the parameters measured during data acquisition from the ones estimated after the CTA and those after the FPA. For the simulation studies an additional superscript 'true' is used to refer to the parameters chosen for simulation data generation and it is the aim of the methods presented to recover these.

\section{METHODOLOGY}

As it will be seen, the proposed tomographic alignment algorithm requires several tomographic reconstructions during operation, which are progressively improved (in terms of spatial 
resolution) during each alignment step. For this reason we start by defining an algebraic tomographic reconstruction algorithm for wrapped phase-contrast data.

\subsection{RECONSTRUCTION OF WRAPPED PHASE-CONTRAST DATA}

In order to account for complex projection geometries an algebraic reconstruction technique, Simultaneous Iterative Reconstruction Technique (SIRT) [32] was extended to wrapped phase-contrast projection data. For simplicity, and because the $\boldsymbol{\Theta}$ values are constant during tomographic reconstruction, we will drop the argument of $\mathbf{A}(\boldsymbol{\Theta})$ and refer to it simply as $\mathbf{A}$. Let us define a residual $\boldsymbol{r}$,

$$
\boldsymbol{r}(\boldsymbol{x})=\boldsymbol{b}-\mathbf{A} \boldsymbol{x} .
$$

The SIRT algorithm [32], [33] is motivated by solving an optimization problem by minimizing a weighted norm of the residual vector $\boldsymbol{r}$ :

$$
\boldsymbol{x}^{\mathbf{R}}=\underset{\boldsymbol{x}}{\operatorname{argmin}}\|\boldsymbol{r}(\boldsymbol{x})\|_{\mathbf{R}}^{2} .
$$

Here $\|\cdot\|_{\mathbf{R}}$ denotes the weighted norm of a vector so that $\|\boldsymbol{x}\|_{\mathbf{R}}^{2}=\boldsymbol{x}^{\mathbf{T}} \mathbf{R} \boldsymbol{x}$. This problem is solved by an iterative algorithm that updates the volume reconstruction at each iteration by

$$
\boldsymbol{x}_{k+1}=\boldsymbol{x}_{k}+\mathbf{C A}^{\mathrm{T}} \mathbf{R} \boldsymbol{r}\left(\boldsymbol{x}_{k}\right),
$$

where $k$ represents the iteration index and $\mathbf{A}^{\mathrm{T}}$ is the transpose of the matrix A. $\mathbf{C}$ and $\mathbf{R}$ are diagonal matrices that contain the inverse of the sum of the columns and rows of the system matrix, so $\mathbf{C}_{j j}=1 / \sum_{i} \mathbf{A}_{i j}$ and $\mathbf{R}_{i i}=1 / \sum_{j} \mathbf{A}_{i j}$. These matrices compensate for the number of rays that hit each pixel and the number of pixels that are hit by each ray.

As the number of iterations go to infinity the iterates will converge to $\boldsymbol{x}^{\mathbf{R}}$, however due to ill-conditioning of the problem, this solution is typically not useful due to excessive noise. The SIRT algorithm exhibits a so-called semiconvergence behavior [34] moving from smooth to increasingly well-resolved but also noisy images, and the best trade-off between resolution and noise is normally obtained by terminating after a finite number of iterations [16], [35], [36].

To take wrapping in the acquired projections $\boldsymbol{b}^{\mathrm{MEA}}$ into account, the residual $\boldsymbol{r}$ computation should be executed in the phase complex domain. This operation is implemented by using the function $Q(f)$, defined as

$$
Q(f)=\arg (\exp (\mathbf{i} f)),
$$

and thus, the modified residual vector $\tilde{\boldsymbol{r}}$ is computed as

$$
\tilde{\boldsymbol{r}}=Q(\boldsymbol{r}) \text {. }
$$

By using $\tilde{\boldsymbol{r}}$ instead of $\boldsymbol{r}$ we have found that this modified SIRT algorithm can return accurate volume reconstructions in the presence of wrapped phase-contrast projection data. We will refer to this modified SIRT algorithm formulation as phase-SIRT.

In this work, we ran 300 iterations of the phase-SIRT algorithm in every tomographic reconstruction with the exception of the auxiliary reconstructions introduced in Section 3.3 where 10 iterations were used instead. An illustration of the phase-SIRT algorithm reconstruction evolution is presented in Figure 10.

The computation of the residual vector according to (13) allows the extension of model-based iterative reconstruction algorithms [36] to wrapped phase-contrast data within reasonable limitations for real data applications. Specifically, in order for the phase-SIRT to provide accurate quantitative results, the wrapped sections of the projection images must be predominantly non-wrapped, and the maximum difference between the true and wrapped phase must not exceed $2 \pi$. These properties were found through empirical observation based on the success of our reconstructions. We do not yet have a full analysis with conditions for convergence of phase-SIRT, but this is subject of ongoing work.

Although it was not necessary for the presented datasets in this publication, we have found empirically that enforcing an elementwise non-negativity constraint on voxel values within the phase-SIRT algorithm allows for robust handling larger wrapped areas. Other constraints and priors could be introduced to decrease noise or artifacts in the reconstructed volume. We have chosen the SIRT algorithm among other iterative reconstruction tools due to its simple formulation and implementation. An alternative robust iterative reconstruction algorithm for phasecontrast tomography that accounts for noise, phase-ramps and projections outliers is described in [37] and will be considered in our future work where we plan to introduce the modification in the cost-function to account for phase-wrapping.

\subsection{COARSE TRANSLATION ALIGNMENT (CTA)}

Image alignment methods based on cross-correlation measurements can be used for tomography alignment [7], [10], [12], [13], [16] by relying on the assumption that translational displacements between two (angular) consecutive projections are negligible. Although these methods can be considered more robust to the presence of linear or constant background phase terms compared with center-of-mass alignment methods, we have found that phase wrapping can lead to their failure.

In our implementation of the cross-correlation alignment method, and in order to exclude the influence of wrapped areas in the phase-contrast projections, we propose the evaluation of relative displacements between projections, not in the image phase domain, but in its phase-gradient domain. Due to the presence of phase ramps and phase wrapping, two consecutive projections are more likely to have a higher cross-correlation measurement for their phase gradient field than for their wrapped phase values. Working in the phase gradient domain decreases the effect of phase ramps, because these are reduced to a constant background with low influence on cross-correlation measurements.

Let $G$ represent the gradient field of $\bar{P}(x, y)$ computed as

$$
G=\sqrt{G_{x}^{2}+G_{y}^{2}},
$$

where $G_{x}$ and $G_{y}$ may be computed by forward or central differences method. Gradient computations will be distorted by discontinuities introduced by phase wrapping. This problem can be overcome by computing the horizontal and vertical gradient fields in the complex phase domain, making it insensitive to phase wrapping. Forward differences are implemented as

$$
\begin{aligned}
& \frac{\partial \bar{P}(x, y)}{\partial x} \approx G_{x}=Q(\bar{P}(x+1, y)-\bar{P}(x, y)), \\
& \frac{\partial \bar{P}(x, y)}{\partial y} \approx G_{y}=Q(\bar{P}(x, y+1)-\bar{P}(x, y)),
\end{aligned}
$$

where division by the step size has been ignored as a constant scaling factor. Two examples of the projection phase gradient intensity field can be seen in Figure 6 and Figure 14. 
After computing the gradient fields $G$ of the projections, crosscorrelation measurements are used to compute the displacement vector between two consecutive projections, from the first to the last with subpixel accuracy. The coordinates of the highest peak in the cross-correlation matrix, evaluated from two adjacent projections, correspond to the relative horizontal and vertical displacement between the two analyzed images. Cross-correlation alignment of tomographic projections requires as many image displacement computations as number of projections that constitute the full dataset. An efficient subpixel up-sampling method is described in [38]

Due to the periodic properties of the Fourier transform, this method is able to detect displacement values up to half of the image dimensions, which is sufficient for most tomographic datasets. The cumulative sum of the computed displacement vectors up to an index $n$ corresponds to the projection parameters $u^{\mathrm{CTA}}$ and $v^{\mathrm{CTA}}$ which are estimates of the relative linear sampledetector displacement of the $n^{\text {th }}$ projection. Errors in the displacement measurements can be accumulated from this sequential calculation, but subpixel evaluations can decrease this effect. At this moment, only relative displacements between the projections are determined. The global alignment of the projections is performed by using the first and last projection from a full-range tomographic dataset $\left(0^{\circ}\right.$ to $\left.180^{\circ}\right)$ as it is commonly done for center-of-rotation (COR) correction [8], concluding the CTA.

As characteristic of parallel-beam projection images, two projections separated by $180^{\circ}$ are symmetric over the rotation axis direction. The computed horizontal displacement value between the first and last projection (flipped over the rotation axis) is used for center-of-rotation correction by applying half of this computed horizontal displacement to the whole projection dataset. The vertical displacement vector between the first and last projection is linearly and evenly distributed over all the projections that constitute the dataset, considering this overall vertical shift as a consequence of accumulation of errors. Both horizontal and vertical alignment errors that may arise from this algorithm step are later optimized in the fine projection parameters alignment.

Differentiation operations may amplify noise, causing a requirement of good signal to noise ratio in the acquired data in order for this method to succeed. Another required condition for this algorithm to succeed is that the phase gradients computed by (15) and (16) should not be wrapped, or if preferred that the phase difference between two adjacent pixels in a projection should be smaller than $\pi$. The same assumptions are made for the derivative FBP introduced in [27].

\subsection{PHASE-RAMP REMOVAL (PRR)}

Although the CTA can provide, for most tested cases, a good relative alignment of projections, background phase ramps must still be removed before tomographic reconstruction in order to provide accurate quantitative results.

The main adopted strategy for removing linear and constant phase terms in the projection images, PRR, is based on the identification of the surrounding areas outside the sample domain in each projection in which phase ramps are to be estimated.

Different tomographic datasets may differ strongly in contrast, morphology and feature sizes making it hard to develop a universal and robust method for segmentation of projections. Phase ramps and wrapping areas may also interfere with pixel- intensity based segmentation techniques which lead us to an alternative approach.

Instead of applying image segmentation methods to the $\bar{P}(x, y)$ projection datasets, we start by making an auxiliary volume reconstruction from the non-normalized phase-contrast projection data, previously aligned by the CTA. The auxiliary reconstructed volume $\tilde{\boldsymbol{x}}^{\mathrm{CTA}}$ is obtained by means of the phaseSIRT algorithm using 10 iterations, $\boldsymbol{\Theta}=\boldsymbol{\Theta}^{\mathrm{CTA}}$ and $\boldsymbol{b}^{\mathrm{MEA}}=\overline{\boldsymbol{P}}$ in (11). The tomogram $\widetilde{\boldsymbol{x}}^{\mathrm{CTA}}$ should not be mistaken for $\boldsymbol{x}^{\mathrm{CTA}}$ reconstructed with the same projection parameters but using 300 iterations in the phase-SIRT algorithm instead. At this stage, the obtained tomographic reconstruction, $\widetilde{\boldsymbol{x}}^{\mathrm{CTA}}$, is not intended to retrieve an accurate and quantitative reconstruction of the volume, but only to attenuate the effect of phase ramps in the image segmentation operation.

We found that the obtained reconstruction was accurately segmented by simple segmentation methods such as Otsu's method [39] allowing the identification of a support volume that defines the sample domain. Otsu's method can be applied to each independent slice or to the whole reconstructed volume. We have also found that for samples with significant contrast in the axial direction (stratified samples with different materials) a slice-byslice approach is preferred for accurate volume support identification.

The objective of threshold segmentation is to assign a zero value to the air sections that surround the support volume and a nonzero value, such as 1, to the voxels belonging to the volume support domain. In this way, we define our volume support $\boldsymbol{x}^{\mathrm{S}}$ as the binary result from Otsu's segmentation method, applied to $\widetilde{\boldsymbol{x}}^{\mathrm{CTA}}$.

The air sections of the reconstructed volume are then mapped to the tomographic projections domain by creating synthetic projections $\boldsymbol{P}^{\text {air }}$, by means of a discretized Radon transform,

$$
\boldsymbol{P}^{\mathrm{air}}=\mathbf{A}\left(\boldsymbol{\Theta}^{\mathrm{CTA}}\right) \boldsymbol{x}^{\mathrm{S}} \text {. }
$$

As the forward-projection operation is equivalent to computing line integrals over the three-dimensional volume, the resulting synthetic projections have zero-intensity pixel values for areas where the integration path does not intersect the volume support and non-zero values otherwise. Figure 7 shows an example of the auxiliary (non-quantitative) reconstruction for the simulated dataset and its resulting segmentation. The zero-valued regions of the synthetic projections are then defined as air regions, from which to determine the appropriate phase ramp correction.

The identified air regions in the projections could be used in a least-squares data-fitting operation (for example) in order to determine the linear and constant phase terms of the background. Guizar-Sicairos et al. presented a least-squares fitting method that takes possible wrapping of the phase ramps into account [27]. Nevertheless we have found this method to be sensitive to the selected air sections especially when some pixels in $\boldsymbol{P}^{\text {air }}$ are incorrectly assigned as 'air' and act as outliers during the data fitting operation. In order to solve this problem, and improve the robustness of the proposed method, we make use of the projection gradients fields values computed by (15) and (16) in the air regions to determine the linear terms of phase ramps in the horizontal and vertical direction. The median of the computed gradients fields in the air sections is taken to reduce possible uncertainties from the segmentation using Otsu's method, and correspond to the first-order term of the background phase ramp in the horizontal and vertical direction respectively. 
After subtracting the first-order phase term from each acquired projection, the constant phase offset is assigned, as the median pixel intensity value of the air regions, and subtracted from each projection. The resulting dataset, $\boldsymbol{b}^{\mathrm{PRR}}$, is now corrected for the undesired phase ramps characteristic of CDI reconstruction techniques. We will refer to the tomographic reconstruction obtained by applying the phase-SIRT algorithm to $\boldsymbol{b}^{\mathrm{PRR}}$ as $\boldsymbol{x}^{\mathrm{PRR}}$. During the PRR the projection parameters are unaffected and thus $\Theta^{\mathrm{PRR}}=\Theta^{\mathrm{CTA}}$.

\subsection{FINE PROJECTION ALIGNMENT (FPA)}

The fine projection parameters alignment optimization of the full proposed algorithm is based on a projection matching technique [11] also known as projection distance minimization [7], [12], [14], [40], [41] by which discrepancies between the real measured projections and synthetic ones made from the current volume reconstructions are minimized.

The introduction of additional DOFs, including tilt angles (described as $\boldsymbol{\alpha}$ and $\boldsymbol{\beta}$ in Figure 1) has been previously published for electron tomography data [41], with a method analogous to ours. The iterative reconstruction method that we use, allows the inclusion of prior information for regularization, and has not, to the authors' knowledge, been reported before for phase-contrast X-ray tomography.

Under the assumption of moderate, zero-mean and Gaussian distributed noise, the projection-matching algorithm as described in [14], [40] aims to solve the alignment and reconstruction problem formulated as a nonlinear least-squares problem of the form

$$
\min _{\boldsymbol{\Theta}, \boldsymbol{x}} \frac{1}{2}\|\boldsymbol{b}-\mathbf{A}(\boldsymbol{\Theta}) \boldsymbol{x}\|^{2} .
$$

This optimization problem was previously characterized in the literature as difficult to solve due to nonlinear coupling between the unknown 3D object $\boldsymbol{x}$ and the projection parameters $\Theta$ [40] and therefore it is commonly solved in an alternating approach solving for $\boldsymbol{\Theta}$ while holding $\boldsymbol{x}$ fixed and, in an analogous way, solving for $\boldsymbol{x}$ while keeping $\boldsymbol{\Theta}$ fixed. This alternating approach is framed in an outer-iterative process that returns after each outer iteration $\boldsymbol{l}$ an updated estimate of $\boldsymbol{x}$ and $\boldsymbol{\Theta}$. The starting values for $\boldsymbol{x}$ and $\boldsymbol{\Theta}$ are chosen according to the previously recovered values after the CTA and PRR operations so that $\boldsymbol{x}_{0}^{\mathrm{FPA}}=\boldsymbol{x}^{\mathrm{PRR}}$ and $\boldsymbol{\Theta}_{0}^{\mathrm{FPA}}=\boldsymbol{\Theta}^{\mathrm{PRR}}$, while $\boldsymbol{b}=\boldsymbol{b}^{\mathrm{PRR}}$.

In the presence of a current reconstructed volume $x_{l}^{\mathrm{FPA}}$, a refinement of the projection parameters in $\boldsymbol{\Theta}$ during the FPA is performed by solving

$$
\boldsymbol{\Theta}_{l+1}^{\mathrm{FPA}}=\underset{\boldsymbol{\Theta}}{\operatorname{argmin}} \frac{1}{2}\left\|\boldsymbol{b}-\mathbf{A}(\boldsymbol{\Theta}) \boldsymbol{x}_{l}^{\mathrm{FPA}}\right\|^{2}
$$

Once an optimum set of projection parameters, for the current $\boldsymbol{x}_{l}^{\mathrm{FPA}}$, is determined, a tomographic reconstruction is computed as

$$
\boldsymbol{x}_{l+1}^{\mathrm{FPA}}=\underset{\boldsymbol{x}}{\operatorname{argmin}} \frac{1}{2}\left\|\boldsymbol{b}-\mathbf{A}\left(\boldsymbol{\Theta}_{l+1}^{\mathrm{FPA}}\right) \boldsymbol{x}\right\|^{2} .
$$

In our implementation of projection matching, the optimization problem described in (19) was solved via a Levenberg-Marquardt algorithm (LMA) implementation described in this section, whereas the reconstruction problem in (20) was replaced by the phase-SIRT algorithm described in section 3.1. It is important to note that, the SIRT reconstruction algorithm solves a different optimization problem than in (20) by minimizing a weighted norm of the residual vector instead of its 2-norm as in expression (20).

The LMA [42], [43] is a nonlinear least-squares optimization algorithm which has previously shown to yield faster and sometimes better convergence when compared with other similar techniques such as quasi-Newton BFGS [14], Gauss-Newton or steepest-descent [44]. It consists of a gradient-based minimization technique that takes steps in the negative gradient direction of a cost function with step sizes defined as a linear combination of gradient-descent and Gauss-Newton approaches [44].

The suggested implementation of the LMA makes use of some problem-specific properties that allow its extension to large-scale problems in a computationally efficient way. These are related to the sparse properties of the Jacobian matrix that defines the optimization algorithm search direction and step size.

Let us define a cost function $f$ as in (19),

$$
f(\boldsymbol{r})=\frac{1}{2}\|\boldsymbol{r}\|_{2}^{2}=\frac{1}{2} \sum_{i=1}^{N M n_{p}} r_{i}^{2} .
$$

The LMA is also an iterative algorithm and therefore its iterations will be identified with the counter $m$ in what we will refer to as the inner-iterative process.

At each iteration $l$ of the outer-iterative cycle, each inneriteration $m$ updates the projection parameters in $\Theta^{\mathrm{FPA}}$ by taking steps of size $\boldsymbol{\delta}_{m}$ :

$$
\begin{aligned}
& \boldsymbol{\Theta}_{l+1 ; m+1}^{\mathrm{FPA}}=\boldsymbol{\Theta}_{l+1 ; m}^{\mathrm{FPA}}+\boldsymbol{\delta}_{m}, \\
& \boldsymbol{\delta}_{m}=-(\mathbf{H}+\lambda \operatorname{diag}(\mathbf{H}))^{-1} \boldsymbol{\nabla} f,
\end{aligned}
$$

where $\mathbf{H}$ and $\boldsymbol{\nabla} f$ represent the Hessian matrix approximation and gradient of $f$ evaluated at $\boldsymbol{x}_{l}^{\mathrm{FPA}}$ and $\lambda$ is a Lagrange multiplier. While $\lambda$ is updated at every iteration depending on the costfunction minimization success [42], $\mathbf{H}$ and $\boldsymbol{\nabla} f$ are computed as

$$
\begin{aligned}
& \mathbf{H}=\mathbf{J}^{\mathrm{T}} \mathbf{J}, \\
& \boldsymbol{\nabla} f=\mathbf{J}^{\mathrm{T}} \boldsymbol{r}, \\
& \mathbf{J}_{i, j}=\frac{\partial \boldsymbol{r}_{i}}{\partial \boldsymbol{\Theta}_{j}} .
\end{aligned}
$$

The update formula for $\lambda$ used in this work is described in [45]. $\mathrm{J}$ is the Jacobian matrix of the residual formed by its partial derivatives with respect to $\boldsymbol{\Theta}$, and exhibits a characteristic shape for this tomography alignment problem. In our discrete model, the derivatives that compose the Jacobian matrix are computed and approximated through the use of the finite forward differences scheme. For brevity, we present only the formulation of the Jacobian elements corresponding to the $\boldsymbol{\theta}$ parameter optimization,

$$
\mathbf{J}_{i, \boldsymbol{\theta}}=\frac{\partial \boldsymbol{r}_{i}}{\partial \boldsymbol{\theta}}=\frac{\mathbf{A}(\boldsymbol{\theta}+\mathbf{1} h, \boldsymbol{u}, \boldsymbol{v}, \boldsymbol{\alpha}, \boldsymbol{\beta}) \boldsymbol{x}-\mathbf{A}(\boldsymbol{\theta}, \boldsymbol{u}, \boldsymbol{v}, \boldsymbol{\alpha}, \boldsymbol{\beta}) \boldsymbol{x}}{h},
$$

where 1 represents a vector with unit elements and $h$ the Jacobian step size. Similar expressions are used for the remaining projection parameters.

Bleichrodt [7] presented some remarks concerning the choice of a proper Jacobian step size. He stated that irregularities at small scales of the GPU implementation of the cost function, mainly due to floating-point errors and noise in the acquired projections, can compromise accurate calculations of the Jacobian. We observed the same irregularities in the Jacobian computation and empirically found a step size of $h=10^{-3}$ to prevent the issue. 
As each tomographic projection is only a function of its own projection parameters, the full computation of the Jacobian matrix is an extensive and unnecessary operation. The full Jacobian has dimensions $N M n_{p}$ by $5 n_{p}$, where $n_{p}$ represents the number of acquired projections. Considering the residual vector in (9) the Jacobian matrix has a sparse form as represented in Figure 2. The sparse nature of $\mathbf{J}$ decreases the computational memory requirements by a factor of $n_{p}$, which becomes especially convenient for large-scale problems with a large number of projections.

After the projection parameters in $\Theta_{l+1}^{\mathrm{FPA}}$ are optimized a new reconstruction $\boldsymbol{x}_{l+1}^{\mathrm{FPA}}$ is determined and the outer-iteration cycle can be repeated until a desired convergence criterion. In our work, at this stage, a fixed number of iterations are being imposed as a stopping rule in the phase-SIRT algorithm and inner and outer iterative cycles.

A summary flowchart of the proposed alignment algorithm is presented in Figure 3.

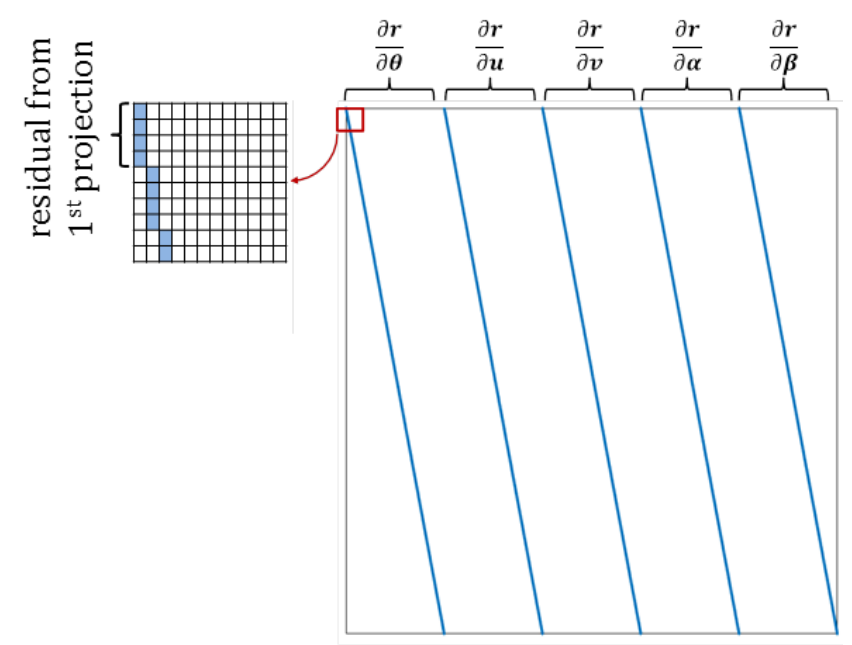

Figure 2: Representation of the characteristic Jacobian structure for the optimization problem with 5 degrees of freedom. The filled (nonblank) lines represent the non-zero values of the Jacobian matrix while the blank areas its null values. In order to easily represent the Jacobian structure highlighted in the magnification, we used a dataset of projections with a total number of pixels $M N=4$.

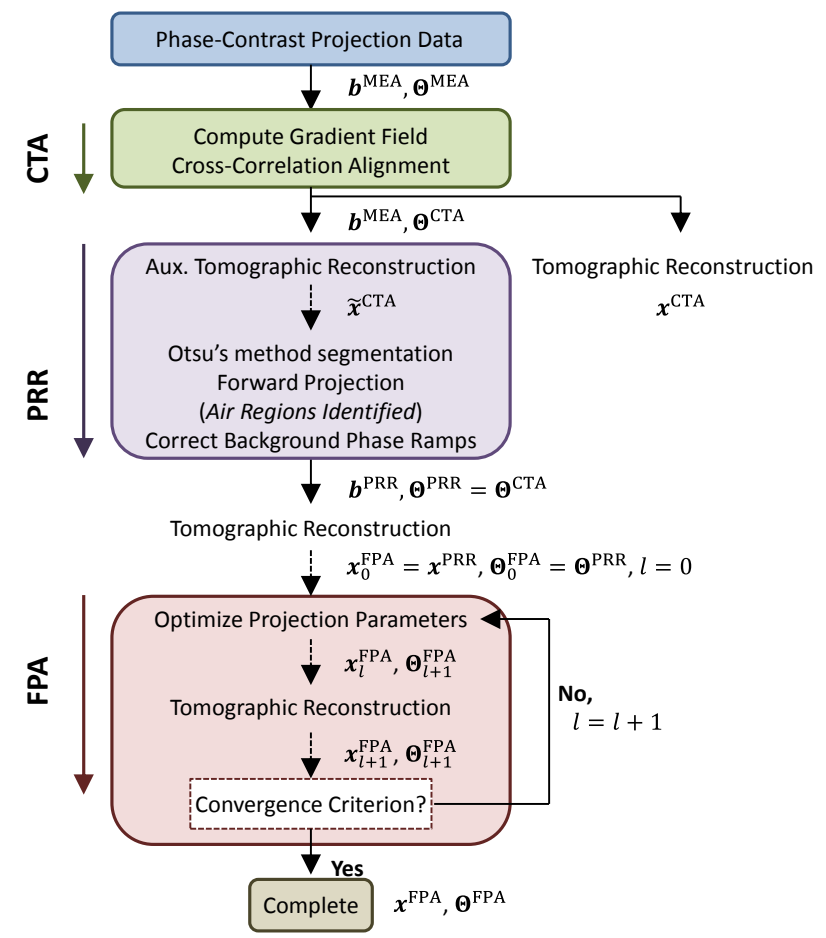

Figure 3: Summary flowchart of the proposed alignment algorithm.

\section{SIMULATION DATA SETUP}

The presented algorithm was developed and tested using MATLAB® 2016a on both Windows and Linux platforms. For both data generation and image reconstruction we made use of the ASTRA toolbox version 1.6 [35], [46] which is an open source, GPU accelerated software platform that allows the full description of the radiation source, sample and detector relative position in space.

As mentioned in section 1, the successive results of the proposed alignment algorithm are assessed by using a 3D modified SheppLogan phantom [47] and real data from a nanoporous glass sample acquired at the cSAXS beamline at Paul Scherrer Institute (PSI), Switzerland. Figure 4 illustrates the 3D modified SheppLogan phantom and the intensity distribution in two different slices.

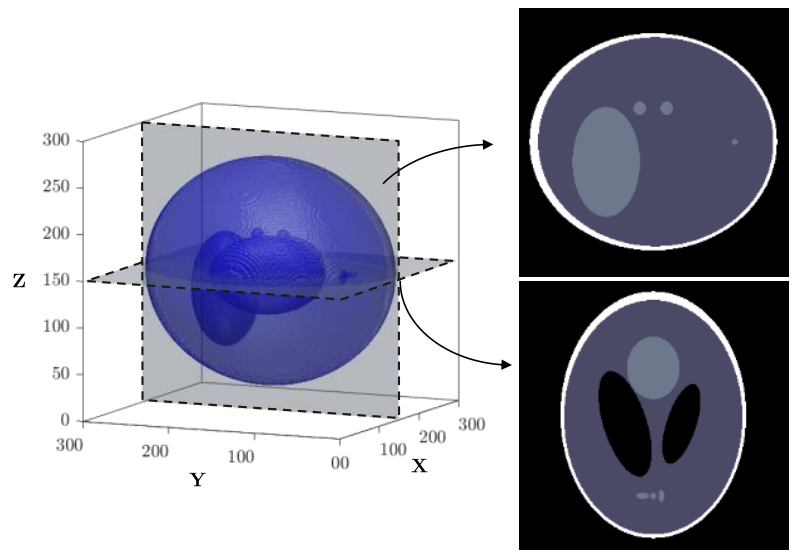

Figure 4: Three-dimensional representation of the modified SheppLogan phantom and intensity maps of two selected slices. 
A 3003-voxel discretized modified 3D Shepp-Logan phantom was used to synthetically produce 360 transmission projections over 180 degrees. In order to simulate mechanical instabilities or position uncertainties, uniform random distributions of the projection parameters described by $\boldsymbol{\theta}, \boldsymbol{u}, \boldsymbol{v}, \boldsymbol{\alpha}, \boldsymbol{\beta}$ were introduced. Such uncertainties were produced within $\left[-1^{\circ}, 1^{\circ}\right]$ for $\boldsymbol{\theta}$, $\left[-5^{\circ}, 5^{\circ}\right]$ for $\boldsymbol{\alpha}$ and $\boldsymbol{\beta}$ and $[-10,10]$ pixels for the linear translations $\boldsymbol{u}$ and $\boldsymbol{v}$. As already mentioned, the superscript 'true' will differentiate the projection parameters used for data generation from the ones recovered through the proposed alignment algorithm. In order to avoid repetitive plots the imposed linear and angular parameters for data generation can be seen in Figure 12 and Figure 13.

To the generated projection images $\boldsymbol{P}^{\text {true }}$, linear background terms were added also with random orientations to replicate the undesired phase ramps. Let us consider $\mathbf{X}$ and $\mathbf{Y}$ as two dimensional matrices with dimensions $N \times M$ so that $\mathbf{X}_{i, j}=j$ and $\mathbf{Y}_{i, j}=i$. Each individual $\operatorname{ramp} S(n)$ for the $n^{\text {th }}$ projection was numerically computed as

$$
S(n)=a(n) \mathbf{X}+b(n) \mathbf{Y}+c(n),
$$

where $a, b$ and $c$ are zero-mean normally distributed random values. For the simulation study presented here, a standard deviation of 0.5 and 0.3 were chosen for the linear terms ( $a$ and $b$ ) and constant term $c$ respectively. The assigned standard deviations produce phase ramps with slopes of the same order of magnitude as those observed in real data measurements.

After generating random background phase ramps, the projections were wrapped according to (30):

$$
\begin{aligned}
& \boldsymbol{P}^{\text {true }}=\mathbf{A}\left(\boldsymbol{\Theta}^{\text {true }}\right) \boldsymbol{x}^{\text {true }}, \\
& \boldsymbol{P}^{\text {wrapped }}=Q\left(\boldsymbol{P}^{\text {true }}+\boldsymbol{S}\right) .
\end{aligned}
$$

\section{QUANTIFICATION OF ALIGNMENT RESULTS}

The attained results from the proposed algorithm were assessed by visual inspection of well-defined features and quantified via Fourier Shell Correlation (FSC) and by monitoring the objective function defined in (15) at the different stages of the proposed algorithm. For the simulation studies an additional relative rootmean-square error (RRMSE) between the reconstructed tomograms and the original phantom was also included.

The FSC is a similarity measure between images or tomograms, often used as a standard resolution criterion for electron microscopy or X-ray tomography, [48]-[50]. It measures the normalized cross-correlation coefficient between two different sub-tomograms over corresponding shells of radius $\bar{r}_{l}$ in Fourier space (see equation (31)). After reconstructing two subtomograms from half the projections dataset each, one can evaluate their agreement by means of the convolution theorem. The FSC function is defined as the summation over all the voxels inside a sphere of radius $r_{i}$ of the normalized cross-correlation in the reciprocal space,

$$
F S C_{f, g}\left(\bar{r}_{i}\right) \stackrel{\text { def }}{=} \frac{\sum_{r=0}^{r_{i}} \boldsymbol{F}^{*}(\bar{r}) \cdot \boldsymbol{G}(\bar{r})}{\sqrt{\sum_{\bar{r}=0}^{\bar{r}_{i}} \boldsymbol{F}^{2}(\bar{r}) \times \sum_{\bar{r}=0}^{\bar{r}_{i}} \boldsymbol{G}^{2}(\bar{r})}},
$$

where $f$ and $g$ are in this case the two mentioned sub-tomograms. $\boldsymbol{F}(\bar{r})$ is the three-dimensional discrete Fourier transform of $f$ at radial position $\bar{r}$ in the reciprocal domain and $\boldsymbol{F}^{*}(\bar{r})$ represents the complex conjugate of $\boldsymbol{F}(\bar{r})$. The chosen frequency threshold is, as suggested by van Heel and Schatz [50], the "1/2-bit" threshold curve defined as

$$
T_{1 / 2-b i t}\left(\bar{r}_{i}\right) \stackrel{\text { def }}{=} \frac{0.2071+1.9102 / \sqrt{n\left(\bar{r}_{i}\right)}}{1.2071+0.9102 / \sqrt{n\left(\bar{r}_{i}\right)}},
$$

where $n\left(\bar{r}_{i}\right)$ is the number of voxels inside a sphere of radius $\bar{r}_{i}$ of the normalized cross-correlation in the reciprocal space.

The RRMSE for a tomographic reconstruction $\boldsymbol{x}$ relative to the original simulated phantom $\boldsymbol{x}^{\text {true }}$ is defined as

$$
\operatorname{RRMSE}(\boldsymbol{x})=\sqrt{\frac{1}{N^{2} M} \frac{\sum_{i=1}^{N^{2} M}\left(x_{i}-x_{i}^{\text {true }}\right)^{2}}{\sum_{i=1}^{N^{2} M}\left(x_{i}^{\text {true }}\right)^{2}}} .
$$

During the described alignment operations, the determined projection parameters may be related to global rigid body movements, such as translations, between the original phantom and the reconstructed tomograms. As an overall translation does not present a decrease in quality or resolution, a global alignment with the original phantom was performed before computation of the RRMSE. The overall displacement vector was computed using a three-dimensional cross-correlation alignment analogous to the algorithm in [38].

\section{RESULTS}

The following images exhibit the results obtained at each state of the proposed algorithm. We will first present the results obtained at each stage of the proposed alignment and reconstruction algorithm for the Shepp-Logan simulation study followed by the ones for the nanoporous glass sample. We will conclude our analysis with the quantification of the resolution gain for both datasets.

\subsection{SIMULATION RESULTS}

Before any alignment operation or phase ramp removal, an immediate tomographic reconstruction $x^{\mathrm{MEA}}$ has limited resolution and strong artifacts that compromise further sample analysis. A representation of the selected slices from $x^{\mathrm{MEA}}$ at this initial stage can be seen in Figure 5.

Figure 6 shows one of the phase projection images for the simulated dataset. In the exhibited projection image it is possible to observe wrapping even in the background linear term. These effects are attenuated in the computed gradient fields (right) that are used for the CTA.

The cross-correlation alignment was performed with an upsampling factor of 100 in the frequency domain by means of the algorithm described in [38].

The central slice for the auxiliary reconstruction used for the sample volume support delimitation is exhibited in Figure 7 (top). On the top-right, one can see the attained segmentation results of the reconstructed volume. The vectors superimposed are intended to illustrate the effect of a discrete line integration operation over the segmented volume. Synthetic projections are generated from the segmented volumes using $\Theta^{\mathrm{MEA}}$ as projection parameters. The resulting auxiliary projections return a zero value for the regions corresponding to the air surrounding the sample that are later used to determine the linear background phase term (phase ramp) orientation and slope. 
(a)
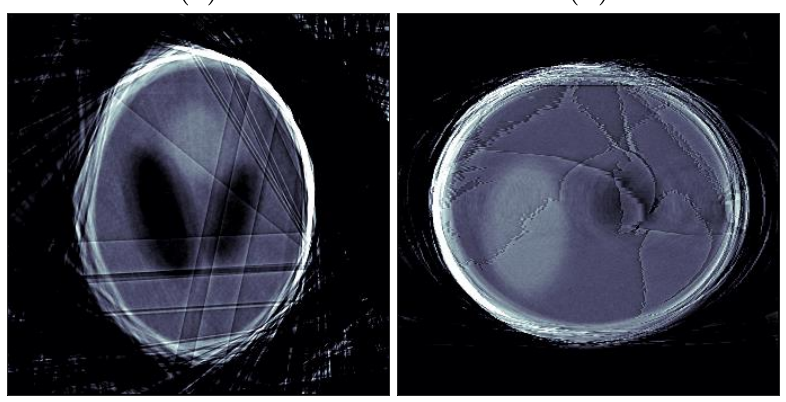

Figure 5: Simulation reconstruction results before alignment and phase ramp removal $\left(x^{\mathrm{MEA}}\right)$ for the two selected slices represented in Figure 4.
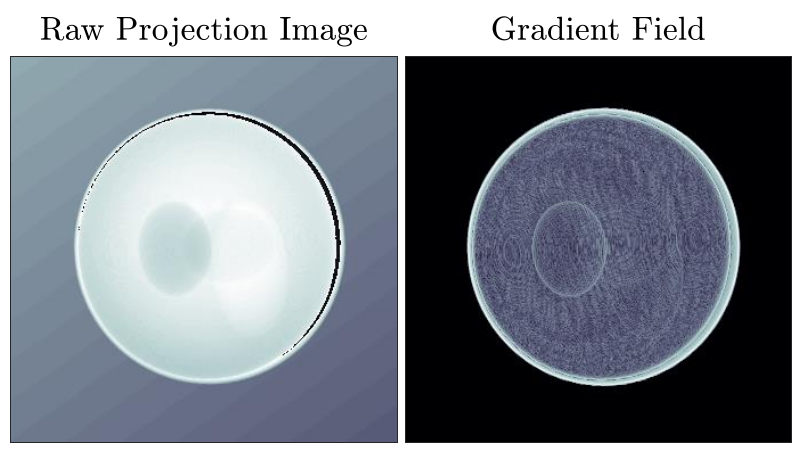

Figure 6: Simulated phase projection image with phase ramp and wrapping $\boldsymbol{b}^{\mathrm{MEA}}$, and respective gradient intensity field computed through (14).
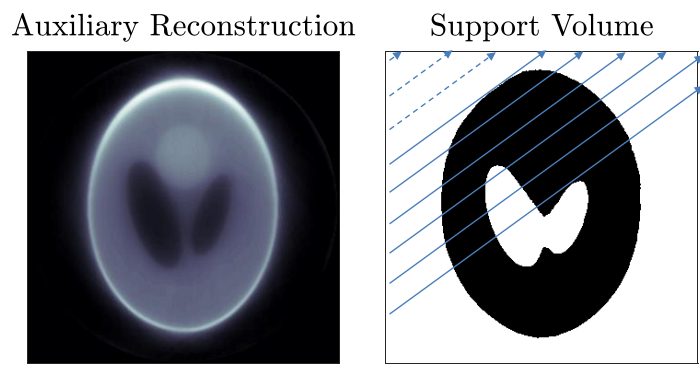

Detected Air Region Ramp Corrected Projection
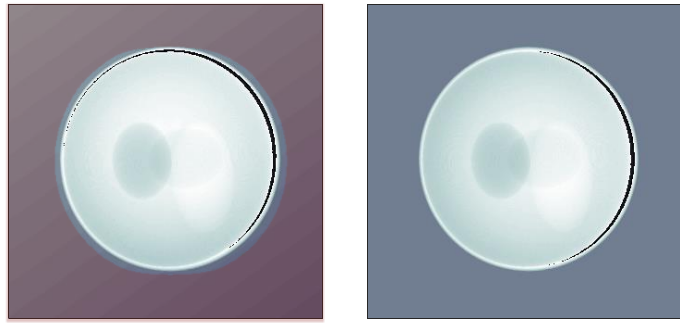

Detected Air Region

Figure 7: On the top: Auxiliary reconstruction $\widetilde{\boldsymbol{x}}^{\mathrm{CTA}}$ used to define the object support volume $\boldsymbol{x}^{\mathrm{S}}$. The effect of phase ramps is attenuated in the object domain. The dashed-line vectors represent areas with no "Xray"-sample interaction that map the air section to the different projections. At the bottom: Simulated projection from $\boldsymbol{b}^{\mathrm{MEA}}$ before PRR and its respective corrected projection from $\boldsymbol{b}^{\mathrm{PRR}}$. Phase wrapping is still present in $\boldsymbol{b}^{\mathrm{PRR}}$ as it is shown in the figure.
The same projection image as in Figure 6 is again represented in Figure 7 (bottom) overlapped with the detected air sections from the previously described method. After the PRR, the phasecontrast projections have been corrected to a zero constant value for the background. It might be worth mentioning that this operation may change the wrapping areas of the projections as it can be seen for the simulation case.

In the FPA, 5 outer iterations were run, with 15 LMA inner iterations and 300 phase-SIRT iterations. The main evolution in the projection parameters estimation was made during the first iterative cycle as it can be seen in Figure 8. The new tomographic reconstructions, represented in Figure 8 by dashed-dot lines, are also responsible for a decrease in the cost function as it can be seen at the end of every 15 iterations.

Figure 9 shows a detailed section of a selected slice of the simulated phantom after PRR, $\boldsymbol{x}^{\mathrm{PRR}}$, and after FPA, $\boldsymbol{x}^{\mathrm{FPA}}$. Resolution is seen to be dramatically increased by FPA.

The proposed modification in the SIRT algorithm allowed the extension of this reconstruction technique for phase-contrast wrapped projection data. During the first iterations, with the previously stated assumption of predominantly non-wrapped projections, some progress toward $\boldsymbol{x}^{\text {true }}$ is achieved.

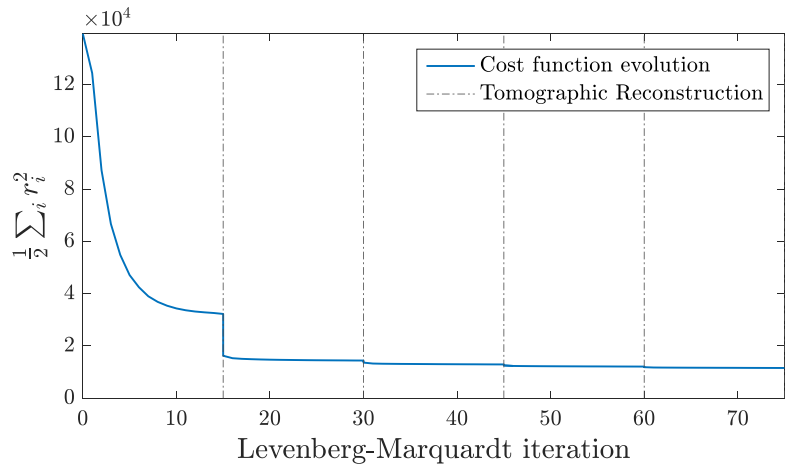

Figure 8: Cost function evolution for the simulation data during FPA. The dashed-dot lines indicate the moment when a new tomographic reconstruction was performed. (a)

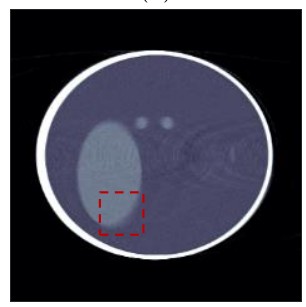

(c)

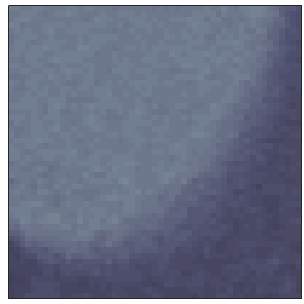

(b)

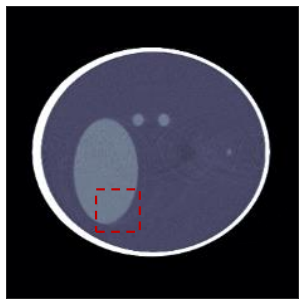

(d)

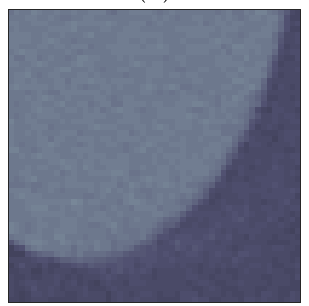

Figure 9: (a) Reconstruction results after PRR $x^{\mathrm{PRR}}$ and (b) after the FPA $\boldsymbol{x}^{\mathrm{FPA}}$. A close inspection of some phantom features exhibits a gain in the reconstructed volume resolution. The regions of interest defined 
by the dashed squares in (a) and (b) are shown in (c) for $\boldsymbol{x}^{\mathrm{PRR}}$ and in (d) for $\boldsymbol{x}^{\mathrm{FPA}}$.

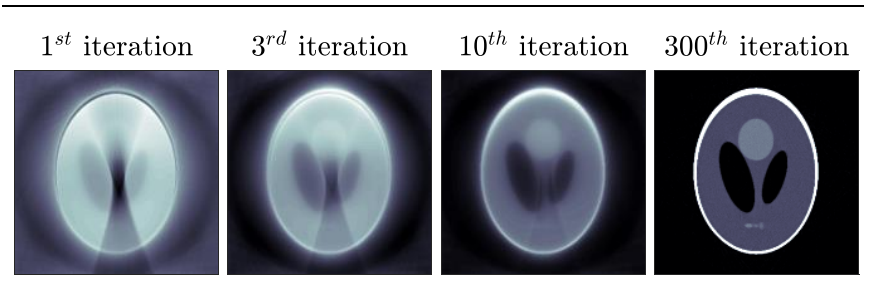

Figure 10: Central slice representation of the final reconstructed tomogram $\boldsymbol{x}^{\mathrm{FPA}}$ over different iterations of the phase-SIRT algorithm.

However, wrapped areas in the phase-contrast projections are responsible for reconstruction artifacts. This effect can be seen in Figure 10 where the central area of the represented slice is highly corrupted by wrapped projections at the start of the tomographic reconstruction. Such artifacts are a consequence of back-projecting an overestimated residual vector in the wrapped-projections areas. As $\boldsymbol{x}_{k}$ is successively updated in each iteration, the phase difference between the forward projections $\mathbf{A}(\boldsymbol{\Theta}) \boldsymbol{x}_{k}$ and $\boldsymbol{b}^{\text {PRR }}$ becomes smaller and is expected to converge to zero.

By computing the residual vector as in (13) we ensure that the back-projected residual vector is not overestimated by $2 \pi$ periods in the wrapped areas of $\boldsymbol{b}^{\mathrm{PRR}}$. This operation constrains the residual vector to a $]-\pi, \pi]$ interval and it can be said to act as an additional regularization to the SIRT reconstruction problem.

The observed increased resolution is supported by the FSC measurements of the reconstructed volumes before and after the FPA. The FSC curves computed after MEA, CTA, PRR and FPA, and the $1 / 2$-bit threshold curve are exhibited in Figure 11.

The intersection values between the FSC curve and the $1 / 2$-bit threshold indicate an increase of the maximum resolved spatial frequency of the respective tomograms. This gain in resolution is sample and problem dependent and should not be taken as a general representation of the algorithm performance.

The linear projection parameters $\boldsymbol{u}^{\text {true }}, \boldsymbol{v}^{\text {true }}, \boldsymbol{u}^{\mathrm{FPA}}$ and $\boldsymbol{v}^{\mathrm{FPA}}$ are presented in Figure 12. It may be relevant to state that $\boldsymbol{v}^{\mathrm{FPA}}$ was plotted after normalization with its mean value for a better comparison with $\boldsymbol{v}^{\text {true }}$. In an analogous way, overall tomogram translations in directions perpendicular to the rotation axis will affect the $\boldsymbol{u}^{\text {FPA }}$ by a sinusoidal function. For the presented simulation study, a global translation of 1 pixel (approximately) in the $y$ direction between $x^{\text {true }}$ and $\boldsymbol{x}^{\mathrm{FPA}}$ affects $\boldsymbol{u}^{\mathrm{FPA}}$ so that $\boldsymbol{u}^{\mathrm{FPA}} \approx \boldsymbol{u}^{\text {true }}+\cos \left(\boldsymbol{\theta}^{\mathrm{FPA}}\right)$. For this reason larger differences between $\boldsymbol{u}^{\text {FPA }}$ and $\boldsymbol{u}^{\text {true }}$ are seen at projection angles closer to $0^{\circ}$ and $180^{\circ}$ and smaller differences at projection angles closer to $90^{\circ}$.

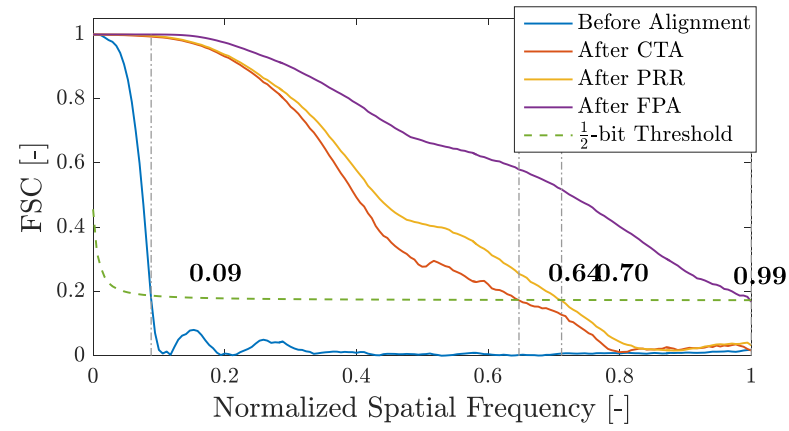

Figure 11: Fourier Shell Correlation measurements for the SheppLogan phantom.

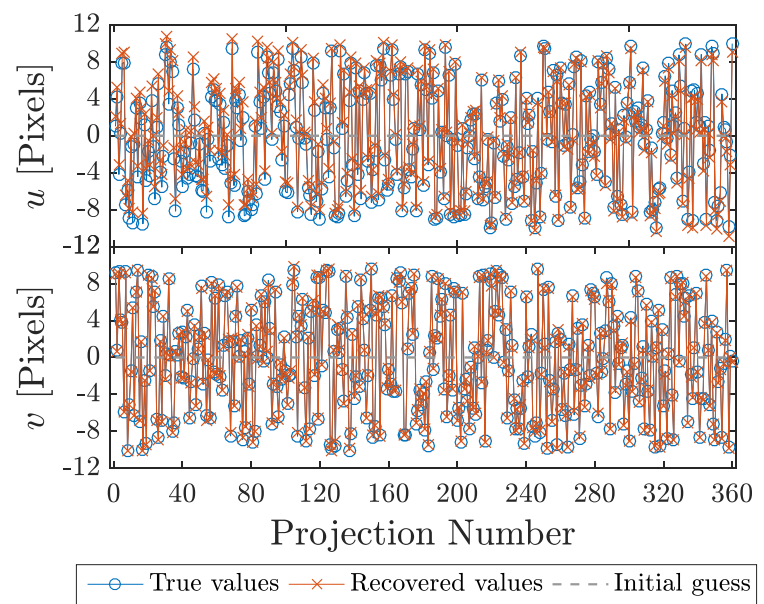

Figure 12: Comparison between $\boldsymbol{u}^{\text {true }}$ and $\boldsymbol{u}^{\text {FPA }}$, and $\boldsymbol{v}^{\text {true }}$ and $\boldsymbol{v}^{\text {FPA }}$ for the 3D Shepp-Logan phantom.

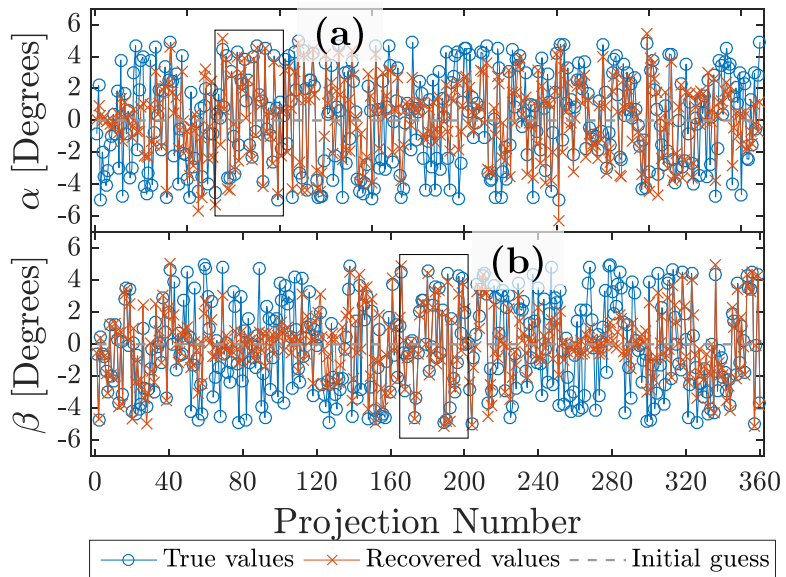

(a)

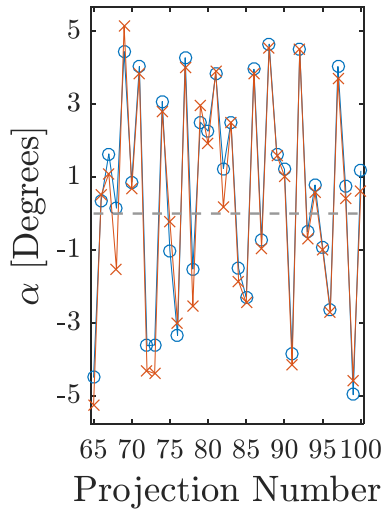

(b)

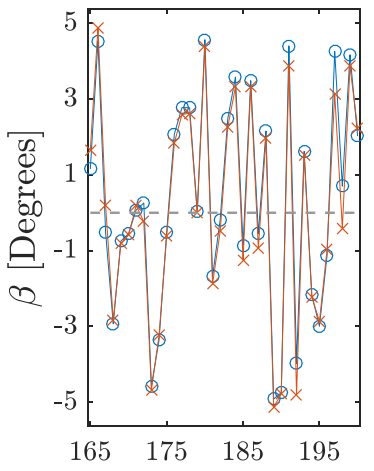

Projection Number
Figure 13: On top: Comparison between $\alpha^{\text {true }}$ and $\alpha^{\mathrm{FPA}}$, and $\beta^{\text {true }}$ and $\beta^{\text {FPA }}$ for the 3D Shepp-Logan phantom. At the bottom: detailed views of the recovered $\boldsymbol{\alpha}$ and $\boldsymbol{\beta}$ parameters with the highest agreement with (a) $\boldsymbol{\alpha}^{\text {true }}$ and (b) $\boldsymbol{\beta}^{\text {true }}$. 
Despite the observed resolution increase, the FPA was only able to fully recover some of the tilt angular parameters $\boldsymbol{\alpha}$ and $\boldsymbol{\beta}$. The recovered values for these last mentioned parameters are presented in Figure 13. A detailed view of the recovered parameters with the highest agreement with $\boldsymbol{\alpha}^{\text {true }}$ and $\boldsymbol{\beta}^{\text {true }}$ is presented at the bottom.

(a)

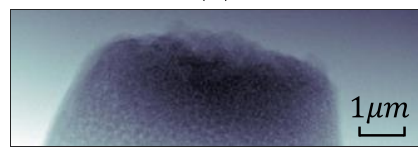

(c)

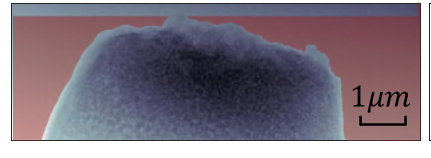

Detected Air Region

Figure 14: (a) Acquired phase-contrast projection; (b) projection gradient field; (c) detected air region and (d) phase-contrast projection after phase ramp removal. The "bright pixels" inside the sample in (b) are due to phase wrapping caused by PRR.

It was not clear from our simulation studies why some of the tilt projection parameters seem to converge to their true values for some of the projections while others do not. We believe that this effect could be related to the ellipsoidal shape of the 3D SheppLogan phantom that makes the LMA more sensitive to the $\boldsymbol{\alpha}$ parameters changes for some of the projections and more sensitive to the $\boldsymbol{\beta}$ parameter for others.

\subsection{REAL DATA RESULTS}

We now present results for the real nanoporous glass dataset. Figure 14 shows an example of a projection image (a), its gradient field (b), the air region detected for PRR (c) and the same projection image after PRR (d). Please note that the bright points in the projection after phase ramp removal (d) are due to phase wrapping that are caused by PRR.

The cost function evolution during FPA for the nanoporous glass sample is presented in Figure 15.

Figure 16 shows the determined $\boldsymbol{u}^{\mathrm{FPA}}, \boldsymbol{v}^{\mathrm{FPA}}, \boldsymbol{\alpha}^{\mathrm{FPA}}$ and $\boldsymbol{\beta}^{\mathrm{FPA}}$ for the nanoporous glass dataset.

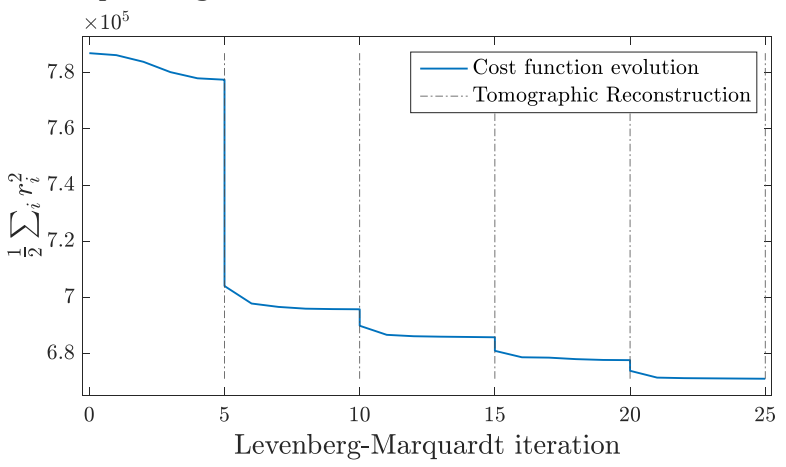

Figure 15: Cost function evolution for the real dataset during FPA. The dashed-dot lines indicate the moment when a new tomographic reconstruction was performed. Please note that the y-axis does not extend to zero in this figure.
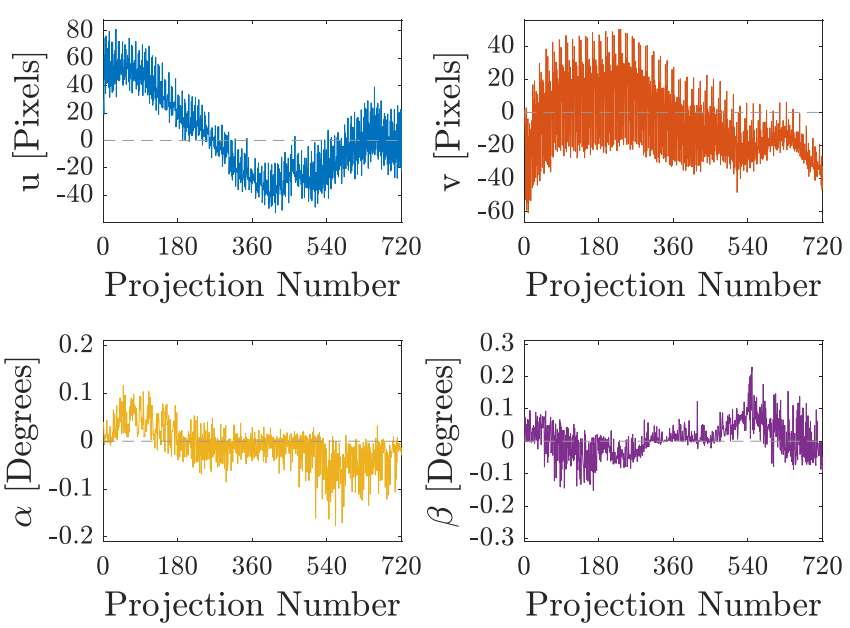

$-\mathrm{u}-\mathrm{v}-\alpha-\beta$

Figure 16: Linear and tilt angular parameters recovered after the fine projection parameters alignment for the nanoporous glass sample. (a)

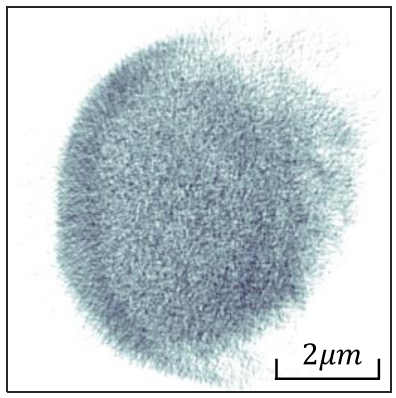

(c)

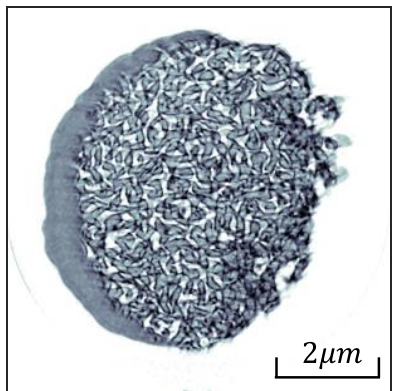

(b)

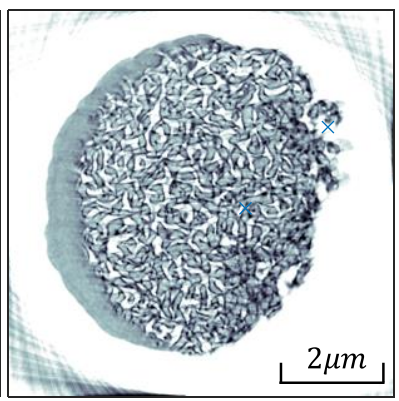

(d)

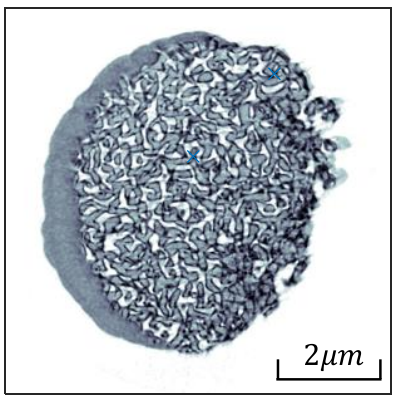

Figure 17: Central slice of the nanoporous glass tomogram before alignment: (a) $x^{\mathrm{MEA}}$, (b) $x^{\mathrm{CTA}}$, (c) $x^{\mathrm{PRR}}$ and (d) $x^{\mathrm{FPA}}$. The tomogram in (b) is not quantitatively accurate since it was reconstructed before PRR. Differences between (c) and (d) are not significantly visible to the naked eye to allow conclusions about possible resolution improvements after the FPA.

The central slices of the reconstructed tomograms at each stage of the full proposed algorithm are presented in Figure 17. As it can be seen, for the nanoporous glass dataset the major resolution gain was achieved by CTA and PRR. This observation is supported by the FSC measurements at the different stages of the proposed algorithm for the nanoporous glass dataset, presented in Figure 18.

No significant improvements in the obtained resolution were observed after FPA for this specific sample. This can possibly be 
explained by the small detected values for $\boldsymbol{\alpha}^{\text {FPA }}$ and $\boldsymbol{\beta}^{\text {FPA }}$ (almost all smaller than $0.1^{\circ}$ ) which indicate that the projections were acquired with a stable (tilt insensitive) rotation stage at the cSAXS beamline at PSI. Nevertheless, the final calculated FSC values for this sample, with a pixel size of $10.2 \mathrm{~nm}$, indicate an isotropic three-dimensional tomographic resolution around $13.6 \mathrm{~nm}(0.75)$ of the Nyquist frequency. This is a $14.5 \%$ improvement compared to the obtained resolution of $15.9 \mathrm{~nm}$ previously reported for the same dataset [31].

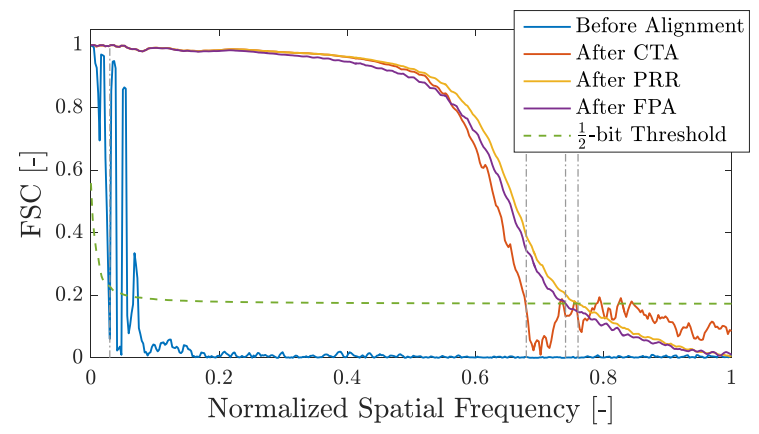

Figure 18: Fourier Shell Correlation measurements for the nanoporous glass sample.

This result suggests that our proposed alignment algorithm can match or slightly improve the resolution obtained by a nonautomated alignment method but, crucially, using a fully automated procedure. Whereas this resolution gain is specific for the dataset and different experimental setups and specimens may behave differently, we expect that similar gains can be achieved under comparable experimental conditions of low-noise and predominantly non-wrapped projections. In the presence of large artifacts, unpaired phase residuals and high noise levels in the tomographic projections, user-assisted algorithms such as the one in [27] may be preferable, but at the cost of intervention by an experienced user. We also note that the reported resolution improvement is not only due to the proposed alignment method but also a consequence of the chosen iterative reconstruction algorithm (phase-SIRT) that we have found more robust to noise than the FBP algorithm applied to the derivative of the wrapped phase used in [31].

In both datasets an increase in the FSC measurements and a decrease in the cost function (21) were observed during the whole proposed alignment algorithm. Table 1 summarizes the results obtained with the different results quantification methods as described in section 4 .

Table 1: Alignment quantification results for both datasets

\begin{tabular}{cccccc}
\hline & & $\begin{array}{c}\text { Before } \\
\text { Alignment }\end{array}$ & $\begin{array}{c}\text { Coarse } \\
\text { Alignment }\end{array}$ & $\begin{array}{c}\text { Phase Ramp } \\
\text { Removal }\end{array}$ & $\begin{array}{c}\text { After Fine } \\
\text { Alignment }\end{array}$ \\
\hline FSC & Simulation & 0.09 & 0.64 & 0.70 & 0.99 \\
& Real Data & 0.03 & 0.68 & 0.76 & 0.75 \\
\hline $\begin{array}{c}\mathbf{1} \\
\sum_{i} r_{i}{ }^{2} \\
\times 10^{4}\end{array}$ & Simulation & 584.4 & 354.1 & 13.95 & 1.14 \\
\hline $\begin{array}{c}\text { RRMSE } \\
\times 10^{-5}\end{array}$ & Simulation & 10.5 & 6.62 & 4.39 & 3.82 \\
\hline
\end{tabular}

\section{CONCLUSIONS \& FUTURE WORK}

The results presented here suggest that the proposed algorithm is able to return accurate tomographic alignments with an automated approach matching resolution values obtained by a non-automated method. This fact makes our alignment and reconstruction algorithm a perfect candidate for phase-contrast tomography data processing, avoiding unnecessary, timeconsuming and subjective user interaction. The automated nature of our algorithm also allows processing of several different datasets simultaneously, which can be especially beneficial to handle ever-increasing data acquisition rates supplied by more brilliant sources. By fully automated we imply that our proposed alignment and reconstruction algorithm is able to run in an unsupervised fashion after an initial set of parameters is defined. These include the up-sampling factor for the cross-correlation calculations, the number of iterations used during tomographic reconstruction, the number of inner and outer iterations in the FPA, and the finite differences step-size during the Jacobian calculation. We found the parameters used in this manuscript to work well for the tested simulations and real data acquired by our research group in the past 3 years with the exception of thick samples.

In the presence of large tilts, as in the simulation study, the FPA was able to recover better estimates of the angular projection parameters contributing to a significant increase in the final attained tomographic resolution. The introduction of the FPA in the real acquired data did not seem to improve the reconstruction accuracy or overall resolution, possibly due to the presence of small and negligible tilt angles during the acquisition of tomographic projections. Nevertheless, the calculated FSC values for the nanoporous glass sample indicated a satisfactory threedimensional tomographic resolution when compared with similar results from the literature [31].

For phase-contrast projection data, we have found that working with the gradient field of projections decreases the effect of undesired phase ramps and phase wrapping during relative alignment of projections.

The SIRT algorithm was extended to phase-wrapped data and demonstrated to work as desired if the modification in the residual vector computation is implemented as we suggest.

Although the computed resolution measure corresponds to dimensions larger than the voxel width, sub-pixel alignment between projections is required for accurate reconstructions.

Due to the non-convexity of (18) one cannot be sure that the FPA will return the global minimum of the problem. This is a general limitation of projection matching algorithms, which is wellestablished in the literature. Nevertheless, projection matching is capable of producing substantial improvements in tomographic alignment and reconstruction, as established by the results, which we find satisfactory from a practical perspective.

In the future, additional studies will be performed in order to optimize the algorithm convergence and reduce the effect of the irregularities found at small scales due to the GPU discrete implementation.

Other algebraic reconstruction algorithms will be implemented for wrapped phase-contrast data. The algorithm in [37] may show promising results in order to decrease reconstruction artifacts, supported by its robustness to outliers in the projections. 
The described computational tools and simulation test scripts have been made publically available and can be accessed in Ref [51].

\section{Acknowledgments}

This research was supported by the Innovation Fund Denmark (Innovationsfonden) within the CINEMA (allianCe for ImagiNg and Modelling of Energy Applications). The work of JSJ was supported by Advanced Grant 291405 'High-Definition Tomography' from the European Research Council. JWA acknowledges the support of the European Research Council (ERC-consolidator-grant 681881).

We would also like to acknowledge the current cSAXS beamline team at PSI, especially to Manuel Guizar-Sicairos, for all the support and suggestions given during this project as well as for establishing the contact with the team responsible for the nanoporous-glass sample preparation. The sample was created by P. Karvinen (PSI) and coated by atomic layer deposition by Elina Färm, Emma Härlönen and Mikko Ritala (Department of Chemistry, University of Helsinki, Finland).

\section{References}

[1] C. G. Schroer, P. Boye, J. M. Feldkamp, J. Patommel, A. Schropp, A. Schwab, S. Stephan, M. Burghammer, S. Schöder, and C. Riekel, "Coherent X-ray diffraction imaging with nanofocused illumination," Physical Review Letters, vol. 101, no. 9, pp. 1-4, 2008.

[2] Y. Takahashi, A. Suzuki, N. Zettsu, Y. Kohmura, Y. Senba, H. Ohashi, K. Yamauchi, and T. Ishikawa, "Towards high-resolution ptychographic x-ray diffraction microscopy," Physical Review $B$ Condensed Matter and Materials Physics, vol. 83, no. 21, pp. 1-5, 2011.

[3] A. Schropp, R. Hoppe, J. Patommel, D. Samberg, F. Seiboth, S. Stephan, G. Wellenreuther, G. Falkenberg, and C. G. Schroer, "Hard x-ray scanning microscopy with coherent radiation: Beyond the resolution of conventional $x$-ray microscopes," Applied Physics Letters, vol. 100, no. 25, pp. 10-13, 2012.

[4] J. Vila-Comamala, A. Diaz, M. Guizar-Sicairos, A. Mantion, C. M. Kewish, A. Menzel, O. Bunk, and C. David, "Characterization of high-resolution diffractive $\mathrm{X}$-ray optics by ptychographic coherent diffractive imaging," Optics Express, vol. 19, no. 22, pp. 21333-21344, 2011.

[5] M. Holler, M. Guizar-Sicairos, E. H. R. Tsai, R. Dinapoli, E. Müller, O. Bunk, J. Raabe, and G. Aeppli, "High-resolution nondestructive three-dimensional imaging of integrated circuits," Nature, vol. 543, no. 7645, pp. 402-406, 2017.

[6] J. C. Fung, W. Liu, W. J. de Ruijter, H. Chen, and C. K. Abbey, "Toward fully automated high-resolution electron tomography," Journal of Structural Biology, vol. 116, no. 1, pp. 181-189, 1996.

[7] F. Bleichrodt and K. J. Batenburg, "Automatic optimization of alignment parameters for tomography datasets," in Image Analysis: 18th Scandinavian Conference, SCIA 2013, Espoo, Finland, June 17-20, 2013. Proceedings, Berlin, Heidelberg: Springer Berlin Heidelberg, 2013, pp. 489-500.

[8] D. J. Azevedo, Stephen G. Schneberk, J. P. Fitch, and H. E. Martz, "Calculation of the rotational centers in computed tomography sinograms," IEEE Transactions on Nuclear Science, vol. 37, no. 4, pp. 1525-1540, 1990.

[9] T. Donath, F. Beckmann, and A. Schreyer, "Automated determination of the center of rotation in tomography data.," Journal of the Optical Society of America. A, Optics, Image Science, and Vision, vol. 23, no. 5, pp. 1048-57, 2006.

[10] R. Guckenberger, "Determination of a common origin in the micrographs," Ultramicroscopy, vol. 9, pp. 167-173, 1982.

[11] Y. Liu, P. A. Penczek, B. F. McEwen, and J. Frank, "A marker-free alignment method for electron tomography," Ultramicroscopy, vol. 58, no. 3-4, pp. 393-402, 1995.

[12] L. Houben and M. Bar Sadan, "Refinement procedure for the image alignment in high-resolution electron tomography," Ultramicroscopy, vol. 111, no. 9-10, pp. 1512-1520, 2011.

[13] S. Brandt, J. Heikkonen, and P. Engelhardt, "Automatic alignment of transmission electron microscope tilt series without fiducial markers.," Journal of Structural Biology, vol. 136, no. 3, pp. 201213, 2001.

[14] C. Yang, E. G. Ng, and P. A. Penczek, "Unified 3-D structure and projection orientation refinement using quasi-Newton algorithm," Journal of Structural Biology, vol. 149, no. 1, pp. 5364, 2005.

[15] P. Fomby and A. J. Cherlin, "Automatic alignment and reconstruction of images for soft X-ray tomography," Journal of Structural Biology, vol. 72, no. 2, pp. 181-204, 2011.

[16] S. S. Brandt, "Markerless alignment in electron tomography," in Electron Tomography: Methods for Three-Dimensional Visualization of Structures in the Cell, J. Frank, ed. (Springer 2006), pp. 187-215.

[17] J. Als-Nielsen and D. McMorrow, Elements of modern X-ray physics, 2nd ed. Hoboken, USA: John Wiley \& Sons, 2011.

[18] J. R. Fienup, "Phase retrieval algorithms: a personal tour [Invited]," Applied Optics, vol. 52, no. 1, pp. 45-56, 2012.

[19] P. Thibault, M. Dierolf, O. Bunk, A. Menzel, and F. Pfeiffer, "Probe retrieval in ptychographic coherent diffractive imaging," Ultramicroscopy, vol. 109, no. 4, pp. 338-343, 2009.

[20] M. Odstrcil, P. Baksh, S. A. Boden, R. Card, J. E. Chad, J. G. Frey, and W. S. Brocklesby, "Ptychographic coherent diffractive imaging with orthogonal probe relaxation," Optics Express, vol. 24, no. 8, pp. 8360-8369, 2016.

[21] A. M. Maiden and J. M. Rodenburg, "An improved ptychographical phase retrieval algorithm for diffractive imaging," Ultramicroscopy, vol. 109, no. 10, pp. 1256-1262, 2009.

[22] M. Guizar-Sicairos and J. R. Fienup, "Phase retrieval with transverse translation diversity: a nonlinear optimization approach," Optics Express, vol. 16, no. 10, pp. 7264-7278, 2008.

[23] J. R. Fienup, "Phase retrieval algorithms: a comparison," Applied Optics, vol. 21, no. 15, pp. 2758-2769, 1982.

[24] V. Elser, "Phase retrieval by iterated projections," Journal of the Optical Society of America A, vol. 20, no. 1, pp. 40-55, 2002.

[25] J. M. Rodenburg, "Ptychography and related diffractive imaging methods," Advances in Imaging and Electron Physics, vol. 150, no. 7, pp. 87-184, 2008.

[26] M. Langer, P. Cloetens, J.-P. Guigay, and F. Peyrin, "Quantitative comparison of direct phase retrieval algorithms in in-line phase tomography.," Medical Physics, vol. 35, no. 10, pp. 4556-4566, 2008.

[27] M. Guizar-Sicairos, A. Diaz, M. Holler, M. S. Lucas, A. Menzel, R. a. Wepf, and O. Bunk, "Phase tomography from x-ray coherent diffractive imaging projections," Optics Express, vol. 19, no. 22, pp. 21345-21357, 2011.

[28] H. A. Z. and C. L. W. Richard M. Goldstein, R. M. Goldstein, H. A. Zebker, and C. L. Werner, "Satellite Radar Interferometry: Twodimensional Phase Unwrapping," Radio Science, vol. 23, no. 4. pp. 713-720, 1988.

[29] V. V. Volkov and Y. Zhu, "Deterministic phase unwrapping in the presence of noise.," Optics Letters, vol. 28, no. 22, pp. 21562158, 2003.

[30] K. C. Tan, T. H. Kim, S. I. Chun, W. J. Shin, and C. W. Mun, "A simple, fast, and robust phase unwrapping method to unwrap MR phase images," IFMBE Proceedings, vol. 21 IFMBE, no. 1, pp. 487-490, 2008.

[31] M. Holler, A. Diaz, M. Guizar-Sicairos, P. Karvinen, E. Färm, E. Härkönen, M. Ritala, A. Menzel, J. Raabe, and O. Bunk, "X-ray ptychographic computed tomography at $16 \mathrm{~nm}$ isotropic 3D resolution," Scientific Reports, vol. 4, p. 3857, 2014.

[32] P. Gilbert, "Iterative methods for the three-dimensional reconstruction of an object from projections," Journal of 
Theoretical Biology, vol. 36, no. 1, pp. 105-117, 1972.

[33] A. C. Kak and M. Slaney, "Algebraic Reconstruction Algorithms," in Principles of Tomographic Imaging, New York, USA: IEEE Press, 1987, pp. 275-296.

[34] P. C. Hansen, Discrete Inverse Problems, 1st ed. Philadelphia: Society for Industrial and Applied Mathematics, 2010.

[35] W. van Aarle, W. J. Palenstijn, J. De Beenhouwer, T. Altantzis, S. Bals, K. J. Batenburg, and J. Sijbers, "The ASTRA Toolbox: A platform for advanced algorithm development in electron tomography.," Ultramicroscopy, vol. 157, pp. 35-47, 2015.

[36] F. E. Boas and D. Fleischmann, "CT artifacts: causes and reduction techniques," Imaging in Medicine, vol. 4, no. 2, pp. 229-240, 2012.

[37] S. V. Venkatakrishnan, M. Farmand, Y. Yu, H. Majidi, K. Van Benthem, S. Marchesini, D. A. Shapiro, and A. Hexemer, "Robust $\mathrm{X}$-ray phase ptycho-tomography," IEEE Signal Processing Letters vol. 23, no. 7, pp. 944-948, 2016.

[38] M. Guizar-Sicairos, S. T. Thurman, and J. R. Fienup, "Efficient subpixel image registration algorithms," Optics Letters, vol. 33, no. 2, pp. 156-158, 2008.

[39] N. Otsu, "A threshold selection method from gray-level histograms," IEEE Transactions on Systems, Man, and Cybernetics, vol. 9, no. 1, pp. 62-66, 1979.

[40] D. Y. Parkinson, C. Knoechel, C. Yang, C. A. Larabell, and M. A. Le Gros, "Automatic alignment and reconstruction of images for soft X-ray tomography," Journal of Structural Biology, vol. 177, no. 2, pp. 259-266, 2012.

[41] V.-D. Tran, M. Moreaud, É. Thiébaut, L. Denis, and J.-M. Becker, "Robust registration of electron tomography projections without fiducial markers," in SPIE, Computational Imaging IX, 2013, vol. 8657, pp. 1-9.

[42] D. W. Marquardt, "An algorithm for least-squares estimation of nonlinear parameters," J. Soc. Ind. Appl. Math., vol. 11, no. 2. pp. 431-441, 1963.

[43] K. Levenberg, "A method for the solution of certain non-linear problems in least squares," SIAM Journal on Scientific Computing, vol. 11, no. 2, pp. 431-441, 1944.

[44] J. Nocedal and S. Wright, Numerical Optimization, 2nd ed. New York, NY, USA: Springer, 2006.

[45] C. Van Loan, "On the method of weighting for equalityconstrained least-squares problems," SIAM Journal on Numerical Analysis, vol. 22, no. 5, pp. 851-864, 1985.

[46] W. van Aarle, W. J. Palenstijn, J. Cant, E. Janssens, F. Bleichrodt, A. Dabravolski, J. De Beenhouwer, K. J. Batenburg, and J. Sijbers, "Fast and flexible X-ray tomography using the ASTRA toolbox," Optics Express, vol. 24, no. 22, pp. 25129-25147, 2016.

[47] M. C. Schabel, "phantom3d.m." (version 1.0) University of Utah Department of Radiology, Salt Lake City, 2005. Available online: [https://www.mathworks.com/matlabcentral/mlcdownloads/downloads/submissions/28496/versions/1/previews/ tomobox/phantom3d.m/index.html?access_key=].

[48] M. Van Heel, "Similarity measures between images," Ultramicroscopy, vol. 21, no. 1, pp. 95-100, 1987.

[49] G. Harauz and M. Van Heel, "Exact filters for general geometry three dimensional reconstruction," Optik, vol. 78, no. 4. pp. 146156, 1986.

[50] M. Van Heel and M. Schatz, "Fourier shell correlation threshold criteria," Journal of Structural Biology, vol. 151, no. 3, pp. 250262, 2005.

[51] T. Ramos, J. S. Jørgensen and J. W. Andersen, "AutoTomoAlign: MATLAB scripts for automatic tomographic alignment and reconstruction of phase-contrast data," zenodo (2017) [retrieved 5 April 2017], http://doi.org/10.5281/zenodo.495122. 